\title{
Adorno humoriste malgré lui
}

Pour R.B.

En 1844, dans les Manuscrits économico-philosophiques, Karl Marx écrit :

Tout ce que l'économiste national te prend en vie et en humanité, il te le remplace par de argent et de la richesse, et tout ce que tu ne peux pas, ton argent le peut : il peut manger, boire, aller au bal, au théâtre ; il connaît l'art, l'érudition, les curiosités historiques, le pouvoir politique ; il peut voyager, il peut te permettre d'acquérir tout cela ; il peut acheter tout cela ; il est la vraie capacité1.

En 1947, dans la Dialectique de la Raison, Theodor W. Adorno et Max Horkheimer écrivent :

L'opération que le schématisme kantien attendait encore des sujets, à savoir rapporter le divers sensible aux concepts fondamentaux, le sujet en est délesté par l'industrie (culturelle). Elle pratique le schématisme comme service rendu au client ${ }^{2}$.

Là où l'argent peut les choses à notre place, un siècle plus tard, l'industrie culturelle pense à notre place. Ce que fait ressortir la contiguïté de ces deux citations, c'est leur rhétorique. Ici, Marx ne décrit pas empiriquement la circulation du capital, ne propose pas un énoncé théorique, il élucide l'équivalent général

Karl Marx, Manuscrits économico-philosophiques de 1844, trad. Franck Fischbach, Éditions Vrin, Paris 2007, p. 180 (traduction modifiée).

2 Theodor W. Adorno et Max Horkheimer, «L'industrie culturelle. L'Aufklärung comme tromperie de masse ", in Dialectique de la raison, trad. Eliane Kaufholz, Éditions Gallimard, Paris 1974, p. 133. Cette traduction rend le propos d'Adorno et de Horkheimer incompréhensible dans la mesure où elle traduit Subjekt par individu, Schematismus par formalisme, etc. Dans toutes les citations ultérieures, ainsi que dans le titre, nous avons modifié la traduction.

* Universität für angewandte Kunst Wien / Université Paris 8 
allégoriquement, geste qu'il réitère et approfondit dans le premier chapitre du Capital consacré au caractère fétiche de la marchandise. De même, dans l'essai de la Dialectique de la Raison de 1947 intitulé « Industrie culturelle. L'Aufklärung comme tromperie des masses », Adorno et Horkheimer ne mènent pas une enquête empirique sur le phénomène de l'industrie culturelle ; ils ne proposent pas davantage une théorie de la « tromperie » propre à la superstructure. À vrai dire, cet essai ne théorise pas tant qu'il n'invente un style. À même son caractère ulcéré, excessif, sa prose manifeste une aversion fascinée pour son objet. Avec ce texte, Adorno et Horkheimer se sont érigés en maîtres d'œuvre virtuoses d'une jouissance de la mélancolie, qui n'a eu de cesse de se propager et de se banaliser. Cette jouissance-là en est venue à se confondre avec le geste critique lui-même. Si invention d'un "style critique » il y a, c'est l'invention d'un style lui-même réifié, dont les ferments agissent chez des philosophes comme Giorgio Agamben, Guy Debord, Bernard Stiegler.

Nommons un premier paradoxe. N'importe quelle lecture de cet essai, même cursive, remarque qu'il n’y va ni d'une description phénoménale « exacte » de l'industrie culturelle, ni d'une exposition de la « vérité » propre à l'aliénation que celle-ci génère. Citons deux occurrences du texte à l'appui de ce constat.

Le bourgeois dont la vie se scinde en vie d'affaires et vie privée, la vie privée en représentations et en intimité, et l'intimité en maussade communauté conjugale et amères consolations procurées par la solitude, brouillé avec lui-même et avec tous les autres, est virtuellement déjà le nazi à la fois enthousiaste et furieux, ou l'habitant des grandes métropoles incapable de concevoir l'amitié autrement que comme “contact social” avec des gens avec lesquels il n’a aucun contact réel.3

Les réactions les plus intimes des hommes envers eux-mêmes ont été à ce point réifiés, que l'idée de ce qui leur est propre ne survit que dans sa forme la plus abstraite : pour eux, personality ne signifie guère plus que les dents blanches, l'absence de taches de transpiration sous les bras l'absence d'émotivité. Et voici le résultat du triomphe de la publicité dans l'industrie culturelle : les consommateurs sont contraints de devenir eux-mêmes ce que sont les produits culturels qu'ils ont percé à jour. ${ }^{4}$

\footnotetext{
3 Ibid., p. 164.

4 Ibid., p. 176.
} 
Si la synonymie du nazi allemand et de l'agent commercial états-unien devait être exacte, nous serions véritablement livrés à une nuit où toutes les vaches sont grises, et où aucune différenciation de la connaissance ne pénètre plus. Et si l'assimilation du consommateur à ses produits avait le statut d'un énoncé de vérité, alors il faudrait en conclure que l'étant réifié est bien tout ce qu'il y a, qu'il n'y a finalement, pour le dire dans un autre lexique, que des corps et des langages, sans même pouvoir y adjoindre la proposition soustractive cruciale, « sinon qu'il y a des vérités ». Il vaut la peine d'insister : concevoir la « révélation » de la réification comme une opération de vérité revient à s'enferrer dans un contresens, à ignorer le postulat adornien : ce n'est que si ce qui est peut être transformé que ce qui est n'est pas tout.

Sur ce dernier point, Adorno et Horkheimer sont d'ailleurs on ne peut plus clairs. Dans la préface d'ensemble de l'ouvrage, ils écrivent : « Nous n'avons pas le moindre doute - et c'est là notre pétition de principe - que dans la société, la liberté est inséparable de l'Aufkärung5. » Ce n'est pas la démonstration de l'imbrication supposément inévitable entre Aufklärung et domination, mais bien l'éclatement de la mesure du "rien de plus que l'étant », qui fait l'enjeu de la Dialectique de la raison. Autant dire que la tendance de l'Aufklärung à se retourner en mythologie n'est saisissable que comme tendance. D'une part parce que le mythe lui-même est déjà Aufklärung, ce qui le rend d'emblée non-identique à lui-même. D'autre part parce que l'historicité du mythe contient en elle-même une possibilité divergente irréductible, celle d'une Aufklärung qui se sépare de la tendance à la domination ${ }^{6}$. Voilà pour l'inscription de l'essai dans les inversions heurtées, asymétriques de l'ouvrage dans son ensemble.

Pour autant, l'essai sur l'industrie culturelle pose un problème immanent supplémentaire, indépendamment de son inclusion dans le livre. Notons-en d'abord quelques traits frappants. Adorno et Horkheimer ne reculent pas devant des images très prosaïques, ici la transpiration sous les bras, mais il y en a bien d'autres. D'où une sorte de schize envoûtante : les auteurs déclarent leur sainte horreur devant le prosaïsme de la culture industrielle en faisant un usage guerrier du prosaïque dans leur attaque de cette même industrie. Plus généra-

Ibid.

Voir à ce propos Alexander Garcia Düttmann, Philosophie der Übertreibung, Suhrkamp, Francfort-sur-le-Main 2004. 
lement, l'effet claustrophobique du texte provient de son absence de questions, qui donne une forte impression d'angoisse ; il embarque immédiatement dans une série d'affirmations défensives sans répit.

Ce n'est pas une enquête, bien que la cascade d'images s'apparente à des morceaux de sociologie sauvage. On ne peut donc ni le réfuter par d'autres enquêtes, ni y chercher un savoir analytique des objets qu'il traite. Cette industrie n'a nullement pour condition la croyance des consommateurs, qui lui succombent alors même qu'ils l'ont percée à jour : la vérité ne saurait y intervenir sur le mode du désillusionnement. Tout cela apparaît d'emblée, et pourtant cet essai a subi une réification permanente, aussi bien dans le sens de l'exactitude que dans celui d'une vérité adossée à la logique de soupçon. Il donne lieu à des exercices aussi vides que répétitifs de désillusionnement, voire de " dénonciation » de l'industrie culturelle, s'élargissant jusqu'aux abstractions réelles qui ne prétendent même plus à une critique, mais seulement à une " cartographie ». Son style d'écriture n'a cessé d'être imité, dégradant peu à peu l'aversion initiale en une figure ornementale, elle aussi disponible à la consommation. Dans les années soixante, « l'adornien » était un idiome couramment parlé en Allemagne, et cet essai en constituait la source principale.

Ces prolongements ne sont pas simplement le fait de contresens. Ils répercutent un embarras de la pensée quant au statut matriciel indémêlable de cet essai. En effet, ce dernier continue à nous déranger alors même qu'il peut paraître caduc sous bien des aspects. Les rapprochements avec le nazisme sont politiquement myopes : il n'y a jamais eu autant de luttes ouvrières aux États-Unis qu'entre 1933 et $1947^{7}$. Aux prises avec son propre présent, le texte tend bien plus à brouiller qu’à clarifier les coordonnées de la séquence historique dont il participe.

Pourtant, les difficultés abordées par les deux philosophes continuent à être les nôtres, même si les paramètres ont drastiquement changé. L'industrie culturelle est une machine qui transforme tous les objets en objets satisfaisant des demandes, que ce soit l'art élevé, les films de télévision, les podcast, les confé-

7 Voir à ce propos, « La lutte des classes aux États-Unis » et « Marx à Detroit », in Mario Tronti, Ouvriers et Capital, trad. Yann Moulier-Boutang, Éditions Christian Bourgois, Paris 1977, ainsi que Mike Davis, City of Quartz, Los Angeles, capitale du futur, trad. Michel Dartevelle, Éditions La Découverte, Paris 2006. 
rences youtube, les images vimeo. Ce que nomme le concept d'industrie culturelle, alors comme maintenant, ce sont ces étranges objets sociaux que sont les œuvres sous condition du capital.

En quoi cette intégration de la culture au régime de la valorisation oblige-t-elle à transformer le concept de critique fondé dans la conscience, et, du moins estce l'hypothèse défendue ici, à abandonner sa détermination comme jugement ? Quelle modification l'industrie culturelle introduit-elle dans l'idéologie, étant donné que celle-là n'a plus vraiment une fonction de justification, mais que sa réalité est déjà sa propre idéologie ? Enfin, l'industrie culturelle ne redouble pas simplement la puissance de l'argent, elle se structure autour d'une prolétarisation aggravée (ou du succès).

Bien sûr, les clivages de la critique ne commencent pas avec l'industrie culturelle, mais bien avec le penseur du jugement lui-même qu'est Kant, se prolongeant dans autant de bifurcations : Marx, Benjamin, Deleuze, etc. Il n'empêche : la confrontation avec ces objets "façadisés ", surrationalisés, insomniaques, qui évincent toute surprise, tuent l'ennui, proscrivent l'échec, tend immanquablement à effondrer la critique, à la rendre strictement superfétatoire. Aux prises avec cette opacité, la critique peut bien entendu elle aussi intégrer l'industrie culturelle, ce qu'elle n'a pas manqué de faire sous les formes du relativisme ou du pluralisme.

Mais l'obstacle demeure : quelle critique serait apte à s'immiscer dans l'industrie culturelle, de manière à en fissurer les effets, à générer des bifurcations dans un sensible séquestré, consensualisé ? La question est d'autant plus prégnante que l'art n'échappe pas à cette tendance. Adorno et Horkheimer ont déjà insisté sur ce point : dès lors que l'art répond à un besoin, que ce soit du grand art ou du divertissement, il est ramené à la formule de l'industrie culturelle. On le sait, ce diagnostic n’a cessé de s’aggraver.

L'essai de 1947 sur l'industrie culturelle fait de la critique de la culture un chantier central de la philosophie, allant de pair avec une transformation de son mode d'écriture. Il y a bien des suites. On peut citer quelques occurrences : Slavoj Žižek, et dans une moindre mesure Alenka Zupančič puisent dans l'industrie culturelle comme dans un régime d'exemples. Fredric Jameson appréhende la fantasy et la science fiction comme deux moments d'une synthèse utopique pos- 
sible. On pourrait tirer là bien d'autres lignes, avec Mark Fischer, Boris Groys, etc. Mais le problème d'une critique de l'industrie culturelle, d'une détermination de son statut reste entier. Retour donc au monument que constitue l'essai d'Adorno et de Horkheimer, quelque soixante-quinze ans après sa première publication.

Comment dégager le ferment problématique de cette matrice ? Plusieurs écueils sont à éviter. Le premier postule une continuité historique avec cet essai aux prises avec les années trente et quarante, et partant à l'hypostasier'. Un second écueil consiste à ontologiser la reproductibilité propre à l'industrie culturelle, à l'assimiler à des manières d'être des choses que sont les artefacts esthétiques, ainsi dans les ontologies plates du réalisme spéculatif : c'est le concept d'industrie culturelle, moins les violences du capitalisme9. Le dernier écueil, si massivement présent qu'il est lui-même devenu un « trésor » de l'industrie culturelle, fait de celle-ci l'un des pôles du mauvais infini qui oppose l'art élevé aux souscultures, et à défendre l'un ou l'autre ${ }^{10}$.

Où, quand et comment se formule le concept d'industrie culturelle ? Son exposition relève d'une pensée attachée à élucider son propre présent, et elle se conçoit depuis le monde des avant-gardes. Dans la perspective adoptée ici, il s'agit d'investir le portrait monolithique qu'en proposent Adorno et Horkheimer pour y réactiver une des fissures, une des tendances divergentes qui y sommeillent. Ce cheminement généalogique s'attache avant tout au style, le style de l'industrie culturelle tel que l'essai le thématise, mais surtout le style de l'essai lui-même. Par ce biais, on espère le retour d'un des spectres qui le hante, et qui est rarement aperçu : son humour noir.

8 C'est ainsi qu'Adorno est mis en jeu par les théoriciens de la valeur, notamment Robert Kurz. Ce dernier emboîte également le pas à son maître en ne distinguant aucunement le champ de la politique de celui de l'industrie culturelle.

9 Voir Roger Pouivet, Philosophie du Rock, Éditions PUF, Paris 2010, ou Tristan Garcia, Forme et objet. Un traité des choses, Éditions PUF, Paris 2011.

10 Ainsi d'Agnès Gayraud, qui se propose de réhabiliter la pop contre Adorno, grand contempteur de la musique légère, alors même que la question d'une légitimité du pop semble définitivement sans objet. Agnès Gayraud, «Dialectique du pop, Éditions La Découverte, coll. « La rue musicale », Paris 2018. 
Le tout premier geste de l'essai consiste précisément à interroger la cohérence implacable du consensus sensible comme production d'un style, production qui oblige à reconsidérer tous les attendus de celui-ci. La traque des effets de style est préférée à toute explicitation empirique concentrée sur les sites réels, mais aussi à toute argumentation. L'empirie n'atteint pas le principe, le pseudo-naturalisme rhétorique de l'argumentation rate l'imbrication de l'artificialité et du truisme propre aux composantes que sont le film, le jazz associé à la musique légère, la radio, le magazine. Saisir la stéréotypie de la culture n'est possible qu'à la dramatiser, à percevoir ses traits hors de la complète banalité qui en constitue la logique. L'essai se condense tout entier en une telle dramatisation ; il noue rhétorique et concept dans un acte d'écriture hérétique à leurs distinctions respectives.

Le renversement dramatique opéré peut d'abord se décrire comme suit. L'industrie culturelle génère la contrainte à la conformité, elle sature le temps de loisir, obturant tout effort de pensée. Etant avant tout consensus sensible, elle constitue la doxa, la monotonie de l'homogénéisation accrue caractéristique de ce que les auteurs appellent le « capitalisme tardif ». Ce commerce de l'adhésion puise de toute évidence dans la logique du vraisemblable inaugurée par la rhétorique aristotélicienne du juste milieu. Or voilà que cette industrie vouée tout uniment à un intermédiaire " optimal » fait l'objet d'une étrange permutation : elle se trouve exposée sous l'aspect de quelque chose d'absolument invraisemblable.

Adorno et Horkheimer présentent l'industrie culturelle telle une totalité surpuissante coïncidant avec sa totalisation, éliminant tout dehors. Sa propagation annule intégralement le sujet de la pensée, se substituant à son activité schématique. Son développement historique augmente encore cette puissance. D’abord inscrite dans le cycle du travail et du loisir qui prépare à retourner au travail, l'industrie culturelle gagne de plus en plus de terrain, jusqu'à en arriver à imposer une volatilisation complète du sens. La chose n'en reste pas à une schématisation standardisée des intrigues, à une facilité d'écoute dont l'évidence abrogerait toute inquiétude. Elle va plus loin, s'attaquant aussi bien à l'insouciance rétive du clown, de Chaplin, des Marx Brothers, à la folie des premiers dessins animés. Leur refus affiché, leurs interruptions loufoques de toutes les médiations sacrificielles divisant la vie en loisir et travail tombent eux aussi sous le couperet du divertissement, pré-jugé, pré-fabriqué, pré-digéré, à l’instar 
du baby food. Loin d'un sens, imposé ou non, ce divertissement se transforme en une série de « signaux » appelant des réactions programmées.

Bref, l'essai sur l'industrie culturelle nous met en présence d'une exagération permanente. Pour en saisir la charge, on peut prendre appui sur la remarque d’Adorno concernant l'exagération dans Minima Moralia :

Le sense of proportion finalement se rapporte à ceci que l'on est censé penser dans les relations de mesure et les ordres de grandeur de la vie qui sont déjà établis. Il faut seulement entendre une fois les représentants endurcis de la clique dominante dire : “cela n'est pas si important”, il faut seulement observer, quand les citoyens parlent d'exagération, d'hystérie, de lubie, pour savoir que c'est exactement à l'endroit où l'appel à la raison est invoquée de la manière plus prompte, que s'annonce une apologie de l'irrationnel. ${ }^{11}$

L'exagération ne provient pas tant de sa mise en jeu rhétorique que d'un rappel des limites, d'une réaction hostile face à la pensée. Tout surcroît de réflexion ne coïncidant plus avec l'état de fait est taxé d'exagération par d'autres. L'effet d'exagération se trouve écartelé, divisé par la socialité qui l'informe. D’une certaine manière, il apparaît comme étant sans rapport avec ce qui le cause. Dans l'essai d'Adorno et de Horkheimer, cette disjonction est reprise à front renversé. Là où, à proprement parler, il n'y aurait rien à penser, puisque le filtrage industriel des sensations a subjugué toutes les irrégularités, impensés, ratages, les philosophes choisissent de s'en étonner.

Une première manière de s'étonner revient à accorder à l'industrie culturelle le crédit d'une invention stylistique, là où les historiens de l'art nostalgiques n'y voient que sa dégradation.

Les historiens de l'art et les défenseurs de la culture n'ont aucune raison de se plaindre de la disparition de l'énergie créatrice du style en Occident. L'utilisation stéréotypée de tout - même de l'informe - en vue de la reproductibilité industrielle, dépasse en rigueur et en valeur tout ce qu'on appelle style, ce concept par lequel tous les amis de la culture idéalisent le passé précapitaliste qu'ils consi-

${ }_{11}$ Theodor. W. Adorno, Minima Moralia, trad. Eliane Kaufholz, Éditions Payot \& Rivages, Paris 2011, p. 89. 
dèrent comme organique. [...] Aucun constructeur du Moyen Âge n’a passé en revue les sujets des vitraux et des sculptures avec plus de suspicion que celle avec laquelle la direction des studios de cinéma examine une œuvre de Balzac ou de Victor Hugo avant de l'accepter définitivement. Aucun théologien médiéval ne pouvait déterminer le degré de tourments à infliger aux damnés conformément à l'ordo de l'amour divin avec un soin plus méticuleux que celui avec lequel la direction d'une superproduction calcule la torture que devra subir le héros, ou la hauteur de l'ourlet de la robe de la vedette. Le catalogue explicite et implicite, exotérique et ésotérique de ce qui est interdit et de ce qui est toléré est si étendu qu'il ne se contente pas seulement de circonscrire le secteur laissé libre, mais qu'il le domine de fond en comble. [...] L'obligation permanente où elle se trouve de produire sans cesse de nouveaux effets qui restent pourtant conformes à l'ancien modèle, sert uniquement, comme une règle supplémentaire, à augmenter le pouvoir des conventions auxquelles chaque effet aurait tendance à échapper. ${ }^{12}$

La continuité stylistique propre aux anciens styles - moyen âge, renaissance configurait l'unité structurante des formes sociales de domination. Mais cette unité n'existait réellement qu'à être parcourue de discordances : l'inscription à même le style d'une promesse qui n'appartient plus à son unité, mais la désagrège. Historiquement, l'activation de cette dialectique contradictoire génère une constellation nouvelle. L'usure moderne de la stabilité traditionnelle du stylistique se renverse en une négation complète de la continuité, notamment chez Dada ou chez les expressionnistes : refus de la convention formelle au profit d'un protocole d'enregistrement direct des affects pour ceux-ci, focalisation "scandaleuse » sur la désagrégation des conventions chez Dada, mettant en jeu les décharges pulsionnelles destructrices, niant toutes les formes préfabriquées d'être, de parler, d'agir, court-circuitant l'œuvre.

Le style de l'industrie culturelle procède en sens inverse de cette dynamique. Il nie tout ce qui du matériau, des gestes, des formes, de la tradition, des techniques ne se prête pas à la convention. D’où sa surrationalité : il n'y a rien en lui qui ne soit programmé, calculé, voulu, adapté au monde tel qu'il va. La négation de toute tension contradictoire entre ses moments a pour effet de les rendre tous interchangeables : le détail peut se substituer au tout, la règle à l'objet spécifique, le particulier au général, etc. Dans la mesure où il supprime toute pola-

${ }_{12}$ Adorno et Horkheimer, Dialectique de la raison, p. 137. 
risation, toute tension des objets qu'il produit, le style de l'industrie culturelle se confond avec la négation du style. Un tel style dispose d'une force prédatrice et intégratrice redoutable, dont les occurrences scandent la longue durée qui nous relie aux années trente : il peut diluer ce qui prétend s’y opposer en le promouvant sur le marché, assimiler n'importe quelle tradition à n’importe quelle autre, réduire l'extériorité dans l'uniformité de sa grille, homogénéiser l'écart entre travail et consommation dans la temporalité du $24 / 7^{13}$.

Quelle que soit sa perspicacité, cette thématisation du style de l'industrie culturelle produit moins l'effet d'un étonnement que celui d'être assommé. Adorno et Horkheimer semblent tellement pris par l'efficace du style que tous deux parcourent qu'ils en oublient de nommer ce qui le troue, ici et là. Certes ils mentionnent, comme en passant, les objets soustraits à la tyrannie du style de l'industrie culturelle : l'indiscipline d'Orson Welles, auquel le studio RKO laissa les mains libres, les films de Chaplin et des Marx Brothers, les premiers dessins animés, Mark Twain. Par contre, ils assortissent ces mentions d'une réserve immédiate : ce ne sont finalement que des alibis qui confirment le système.

C'est faire peu de cas de ceci : l'ensemble de ces objets n'est pensable que sur le terrain de l'industrie culturelle, dans le monde où celle-ci s'impose, selon ses modes de production et distribution, le film, le magazine. Evidemment, ces objets ont une incidence différente, relatives aux distances historiques qui nous en séparent. Sans doute Adorno et Horkheimer étaient-ils marqués par l'élimination de telles possibilités, par l'aplatissement impitoyable que subissait la culture, tandis qu'aujourd'hui nous y percevons davantage des chances inaugurales, une ouverture remise au temps, un possible dans lequel renouveler notre expérience. Dans le contexte des années trente, on pouvait certainement s’arrêter sur la différence que comportait leur archaïsme, évoquer la transition historique entre cirque et film, assigner ces objets à une période " artisanale » de l'industrie culturelle plutôt qu'à sa pleine maturité.

Reste que le rouleau compresseur de l'exagération, barricadé dans la sphère imaginaire d'une toute-puissance du capital, semble incapable de s'interrompre. Disons simplement que sa logique ignore tout des contradictions faisant valoir

${ }_{13}$ Jonathan Crary, 24/7 Le capitalisme à l'assaut du sommeil, trad. Grégoire Chamayou, Éditions La Découverte, Paris 2016. 
les désirs inaliénables contre les savoirs de l'aliénation. À ce titre, cette logique ne fait d'ailleurs que répéter le toujours-semblable de l'exagération triomphale qui caractérise déjà l'industrie culturelle, style que l'on retrouve aujourd'hui dans n'importe quelle quatrième de couverture : « ce livre marquera toute une génération », « ouvrage provocateur, radical, sans concession », etc.

On touche au moment où l'essai fatigue, ennuie, où son sériage et ses arrangements imagés ne cessent de revenir au même point, tout comme dans les films de l'industrie culturelle la fuite pour se marier recolle au quotidien sinistre qu'elle rêve de quitter. Il y a bien là l'impasse d'une économie du soupçon. Mais peut-être faut-il chercher ailleurs le point où l'accumulation des tropes défait cette économie.

Dans l'avancée en cascade du texte, un développement s'esquisse : la mainmise croissante de l'industrie culturelle sur le sujet, le genre humain, l'individu, leur naufrage complet finit par se retourner contre celle-là. En dévorant son propre support, la demande marchande de culture quelle se targue de produire et de satisfaire, en éliminant toujours davantage du client ce qui le différencie encore d'un client, le vacuum de l'industrie culturelle finit par détruire sa propre finalité. Il se transforme alors en une « chose du passé », c'est-à-dire qu'il accomplit sa propre autodissolution.

Vu sous cet angle, l'aspect monolithique de l'essai se charge d'une tension nouvelle. Sa dimension thétique reflue pour laisser place à une dialectisation des extrêmes. Là où l'industrie culturelle prescrit continuellement le fun comme bain vivifiant, Adorno et Horkheimer s'astreignent à la mortifier. L'on se trouve en présence d'un geste critique emprunté tant à Hegel qu'aux surréalistes, celui d'un " humour objectif », qui convertit l'expérience subjective en l'objectivité fantastique d'une hallucination.

"L'industrie culturelle, l'Aufklärung comme tromperie des masses » est-il un monument d'humour noir ? Pour percevoir « ce bord de néant» qui accompagne tous ses développements, il suffit de déporter l'attention de son caractère systématique, assertorique vers son montage. On peut alors se tourner différemment vers la remarque inaugurale : «L'opération que le schématisme kantien attendait encore des sujets, à savoir rapporter le divers sensible aux concepts 
fondamentaux, le sujet en est délesté par l'industrie (culturelle). Elle pratique le schématisme comme service rendu au client $\mathrm{t}^{14}$.»

La citation procède par capillarité ; la doctrine transcendantale kantienne s'étend de manière tordue : elle se dilate en une forme parodique de théorie. Sa conceptualité rigoureuse a été contaminée par le lexique de l'industrie culturelle, sa pureté est compromise. Autant dire que la philosophie ne peut plus simplement se fier à cet intermédiaire élaboré par Kant, à cette synthèse qui faisait du jugement, et donc de la pensée, une activité productrice, un nouage temporel apte à unifier réceptivité du sensible et spontanéité de l'entendement. L'homogénéisation synthétique est tombée aux mains de l'homogénéité industrielle, le coude temporel discontinu rapportant le Je au Moi a été raccourci jusqu'à les amalgamer, et partant à les liquider. Qu'à cela ne tienne : on se passera désormais de l'homogénéisation propre à l'imagination, on ira puiser les agencements du sensible et de l'intelligible ailleurs, notamment dans les procédures mises à disposition par les avant-gardes, la psychanalyse, voire par l'industrie culturelle elle-même, c'est-à-dire par le langage de la publicité. Disloquer et assembler dans l'élément de l'hétérogène, décentrer, soustraire les morceaux du scénario culturel à leur cohérence, défier tous les réquisits de la vérification, improviser des connexions witzig en lieu et place de tout ordonnancement. Voilà autant d'échantillons de méthode que charrie le montage de cet essai.

Celui-ci n'hésite pas à promener ses observations de registre en registre, sans égard pour leur hiérarchisation ou leur localisation. Ainsi, l'on navigue du " sein dans le sweater » à " la star copie d'elle-même », aux " autos et cigarettes » jusqu'à « l'homme sans scrupule », à « la jeune fille dynamique ». Des objets de pulsions partielles côtoient des simulacres, des idéologies-marchandises prennent place à côté d'indications didascaliques : l'industrie culturelle est mise en lambeaux, lambeaux des discours psychanalytiques, des typologies, des anciens arts libéraux, des technologies à partir desquels elle tisse sa toile. Son principe d'unification étant en même temps le principe de sa ruine, sa cohérence étant son irrationalité, il suffit de braquer les projecteurs sur ses processus pour que sa représentation se déchire d'elle-même, pour qu'elle s'identifie étrangement à son propre recadrage, un recadrage interlope, grinçant.

14 Adorno et Horkheimer, Dialectique de la raison, p. 133. 
Dans cet essai, tout apparaît comme reconnaissable et pourtant non familier ; plus rien n'est à sa place, on a passé un seuil sans que l'on soit vraiment passé ailleurs (Brecht n'est pas loin). Adorno et Horkheimer ont beau réprouver le rire comme étant une fuite devant le pouvoir, ils ont beau affirmer que Baudelaire lui-même était dépourvu d'humour, rien n'y fait : là où leur texte touche juste, il déclenche l'hilarité. Sans doute, ces philosophes se tiennent en retrait. Mais à tout le moins, leurs considérations provoquent le rire sans y participer.

Leur maniement corrosif du sérieux sociologique balance d'un coup par-dessus bord les faits, les classes sociales, la géographie, les hiérarchies artistiques, amalgamant l'Europe et les États-Unis, mêlant l'invocation matérialiste de la poudre de pudding aux considérations sur l'incidence des statistiques ${ }^{15}$. Au monde de l'industrie culturelle quadrillé par le préjugé d'exactitude de la connaissance positiviste, l'essai oppose un monde en lequel l'homme a disparu et où les objets, étrangement animalisés par les circonstances, ne cessent de permuter entre différents comportements, tantôt se faisant rébus, tantôt allégorie. Ce démontage hilarant de la platitude positiviste, laquelle affecte aussi bien nombre d'orientations qui prétendent s'en exempter, ainsi de l'objectivisme marxiste ou de la micrologie ethnographique, constitue aujourd'hui plus que jamais un antidote à la bêtise savante des sciences sociales.

Et qu'en est-il du rapport à la vérité ? Les deux philosophes s'en prennent à toute conception du sujet qui prétend fonder l'origine de la conscience dans sa propre activité, et ce faisant manque à penser la façon dont les sujets sont constitués sur le sol de l'objectivité. C'est dans ce contexte qu'ils mettent en avant la défaite infligée par l'industrie culturelle au schématisme kantien.

La chose mérite d'être dépliée, car il n'est rien moins qu'évident que le schématisme tombe sous ce verdict. L’activité productrice qui synthétise réceptivité du sensible et entendement catégoriel génère bien une unité, mais seulement au prix d'y inscrire une non-coïncidence à soi du sujet. En mettant fin aux conceptions contemplatives de l'idée, que celle-ci soit transcendante ou innée, en faisant du jugement synthétique une activité productrice, Kant a découvert un

15 En écho à la célèbre remarque de Friedrich Engels, « la preuve du pudding c’est qu’on le mange ». 
nouveau problème : celui de l'écart irréductible entre le procès d'unification de l'expérience et l'unité qui en résulte.

Rappelons brièvement les termes de ce problème. Le dualisme du réceptif et de l'intelligible donne lieu à un nouage, un intermédiaire : c'est là qu'intervient le schématisme, associé par Kant à la forme a priori du sens interne, le temps. Avec les catégories, qui sont des concepts a priori, hors de l'expérience, la forme du temps a en commun qu'elle est une règle générale. Avec la sensibilité réceptive, elle a en commun qu'elle est contenue dans toute représentation du divers sensible.

La faculté productrice du schème est l'imagination, l'activité de former des images ou de présentifier un non-être, de se donner une vision intuitive de ce qui, « absent », ne relève pas de l'intuition. Cette faculté est bifide. Elle est réceptive dans la mesure où elle ouvre à une intuition, mais cette intuition est créée de toutes pièces, schématisée. Par là, elle est donc active, à l'instar des catégories intellectuelles. Point d'importance : le schématisme n'est pas une image calquée sur des images sensibles, reproduction d'un existant. Se décollant de toute image donnée, toute expérience, les schèmes ne sont des images de rien, mais l'activité temporalisante à partir de laquelle se forment les images.

À proprement parler, l'imagination ne produit rien d'autre que la relation impliquée par la forme du temps. En effet, le temps ne peut être série des maintenant que si ces derniers sont toujours déjà dépassés par une vision unitaire de la série. C’est-à-dire que le temps doit toujours se donner l'image de son propre déploiement pour que le «maintenant » ne s'éparpille pas, ne se perde pas. Il faut qu'il présentifie, à même chaque « maintenant », le schème de tout « maintenant », à savoir le passé, le futur, la série comme règle totalisante.

Ce que génère ainsi le schématisme, c'est une asynchronie du temps, qui fait que je ne peux jamais me retourner sur lui pour le saisir, et surtout que je ne peux jamais me retourner sur moi, que l'objectivation de mon moi est impossible. Dès que je me retourne sur le temps pour m’identifier à moi-même, l'acte de ce retour lui-même échappe. Il y a bien un procès d'unification, mais l'unité de la conscience qu'elle génère se diffracte en même qu'elle se produit. L'unité de la conscience arrive toujours trop tôt ou trop tard dans la conscience qu'elle prend d'elle-même. Ainsi, le schématisme de l'imagination kantienne ne surmonte le 
dualisme qu'en affectant la pensée d'un impensable, en séparant le « je pense » du moi existentiel par la ligne du temps qui les rapporte l'une à l'autre sous la condition d'une différence fondamentale.

On trouve donc bien dans le schématisme transcendantal un clivage inaugural qui affecte irrémédiablement l'unité du sujet moderne. Plus précisément, c'est ce clivage qui constitue le sujet. À partir de là, Adorno et Horkheimer font le constat suivant. La capture active qu'exerce l'industrie culturelle sur l'effort schématisant exigé du sujet, son effacement de l'impensable qui l'affecte en révèle un écueil, atteste d' un «malaise du schématisme ».

Le schématisme kantien temporalise les catégories, les rend applicables aux phénomènes, mais le système catégoriel lui-même conserve un statut invariant, ce qui bloque toute possibilité d'historiciser tant ces catégories transcendantales elles-mêmes, que aussi et surtout l'expérimentation de leurs enchaînements. Il apparaît alors que le tableau catégoriel n'a rien de neutre. Ce qui est conçu par Kant comme condition a priori de l'entendement renvoie en réalité à la genèse de la subjectivité dans le champ objectif du capital.

Que faire de cette hétéronomie du catégoriel ? Faut-il en prendre acte comme d'une aliénation irrévocable, quitte à se consacrer inlassablement à en faire le procès ? Ce penchant n'est pas complètement absent chez Adorno et Horkheimer, il y figure comme une tentation à laquelle les auteurs résistent, mais qu'ils ne surmonteront jamais tout à fait. La force de cet essai réside alors précisément dans la manière inédite qu'il a de déjouer cette conclusion.

Prendre acte, enfin, de ce que le sujet dit « autonome » est toujours déjà un sujet aliéné, cela permet plusieurs déplacements. Le premier est simplement de ne plus chercher l'issue à l'aliénation dans une supposée réappropriation, laquelle ne fait que nous assujettir toujours davantage à l'objectivité déjà donnée. Le second, plus essentiel, est d'ouvrir une voie autre au sein même de l'objectivité.

En effet, qu'il appartienne au sujet d'être aussi un objet, cela ne le condamne pas à l'aliénation. Bien au contraire, cela lui offre tout autant la chance de se décentrer de lui-même : la désorganisation de sa constitution catégorielle lui permet d'échapper enfin à l'imaginaire de son moi, d'expérimenter des modalités non-catégorielles de sa propre extériorité. Ce frayage hétérogène, disparate, 
de la multiplicité " pré-intégrée » par l'industrie culturelle - montage, collage, fragmentation pulsionnelle, sismographie, trognon de jouissance, allégorisation - pose les jalons d'une pensée autre du sujet, d'un sujet auquel le primat de l'objet est accessible, et dont la Dialectique négative proposera une formulation définitive ${ }^{16}$.

Sans doute est-ce ce frayage qui fait tout le prix de l'essai, bien qu'Adorno luimême n'aide pas toujours à discerner cette ligne forte. Ainsi, dans son « Résumé sur l'industrie culturelle ", il s'effraie lui-même des conclusions appelées par cette trajectoire, à savoir que la réalité axiale du sujet n'est aucunement dans son moi, et se rabat une nouvelle fois sur la fonction imaginaire, selon des catégories empruntées directement à l'ego psychology : l'emprise de l'industrie culturelle renverrait à une « faiblesse » du moi, à laquelle il n'y a plus alors qu'à opposer un « moi fort $»^{17}$.

Se vérifie alors, avec et contre les auteurs de «L'industrie culturelle. Aufklärung et tromperie de masse ", tout l'intérêt d'éclairer l'humour qui s'est glissé en contrebande dans leur essai. Car l'humour jamais ne s'arrête en chemin, même quand le chemin mène plus bas. Indifférent aux sirènes de la mélancolie, rétif à toutes les tranquillités que procure le systématique, fussent-elles négatives, plus prompt à dissoudre le moi qu'à y chercher refuge, toujours l'humour explose le cadre dans lequel il s'insère, en descendant dans les conséquences...

\section{Références}

Adorno, Theodor W., Dialectique négative, trad. Groupe de traduction du Collège de philosophie, Éditions Payot, Paris 2003

16 Sujet, Adorno est on ne peut plus clair là-dessus, qui n'est justement pas au-delà de toute objectivation : «C'est seulement parce que le sujet est médiatisé de son côté et donc n'est pas le radicalement autre de l'objet qui seul légitime celui-ci, qu'il est capable de saisir l'objectivité en général ». Theodor W. Adorno, Dialectique négative, trad. Groupe de traduction du Collège de philosophie, Éditions Payot, Paris 2003, p. 147.

${ }_{17}$ Ce recours au « moi fort », qui n'est pas isolé dans son œuvre, ne laisse pas d'étonner chez un penseur comme Adorno. Il invite à reconsidérer le non-rapport de deux penseurs qui ont le même âge, mais n’appartiennent pas à la même époque, Adorno et Lacan. Pour une critique de la notion d'un moi supposément fort ou faible, pour le rapport à la suggestion que cela implique, voir Jacques Lacan, Le Séminaire, livre II, Le moi dans la théorie de Freud et dans la technique de la psychanalyse, Éditions du Seuil, Paris 1977, p. 54 et suiv. 
- Minima Moralia, trad. Eliane Kaufholz, Éditions Payot \& Rivages, Paris 2011

Adorno, Theodor W. et Max Horkheimer, "L'industrie culturelle. L'Aufklärung comme tromperie de masse », in Dialectique de la raison, trad. Eliane Kaufholz, Éditions Gallimard, Paris 1974

Crary, Jonathan, 24/7 Le capitalisme à l'assaut du sommeil, trad. Grégoire Chamayou, Éditions La Découverte, Paris 2016

Davis, Mike, City of Quartz, Los Angeles, capitale du futur, trad. Michel Dartevelle, Éditions La Découverte, Paris 2006

Düttmann, Alexander Garcia, Philosophie der Übertreibung, Suhrkamp, Francfort-sur-leMain 2004

Garcia, Tristan, Forme et objet. Un traité des choses, Éditions PUF, Paris 2011

Gayraud, Agnès, Dialectique du pop, Éditions La Découverte, coll. « La rue musicale », Paris 2018

Marx, Karl, Manuscrits économico-philosophiques de 1844, trad. Franck Fischbach, Éditions Vrin, Paris 2007

Lacan, Jacques, Le Séminaire, livre II, Le moi dans la théorie de Freud et dans la technique de la psychanalyse, Éditions du Seuil, Paris 1977

Pouivet, Roger, Philosophie du Rock, Éditions PUF, Paris 2010

Tronti, Mario, Ouvriers et Capital, trad. Yann Moulier-Boutang, Éditions Christian Bourgois, Paris 1977 